\title{
A search for possible interactions between ejections from GRS 1915+105 and the surrounding interstellar medium
}

\author{
S. Chaty ${ }^{1,2}$, L. F. Rodríguez ${ }^{3}$, I. F. Mirabel ${ }^{2,4}$, T. R. Geballe ${ }^{5}$, Y. Fuchs ${ }^{2}$, A. Claret $^{2}$, \\ C. J. Cesarsky ${ }^{6}$, and D. Cesarsky 7,8
}

1 Department of Physics and Astronomy, The Open University, Walton Hall, Milton Keynes, MK7 6AA, UK

2 Service d'Astrophysique, DSM/DAPNIA/SAp, CEA/Saclay, L'Orme des Merisiers, Bât. 709, 91191 Gif-sur-Yvette Cedex, France

3 Instituto de Astronomía, UNAM, Campus Morelia, Morelia, Michoacán 58190, Mexico

4 Instituto de Astronomía y Física del Espacio C.C. 67, Suc. 28. 1428, Buenos Aires, Argentina

5 Gemini Observatory, 670 N. A'ohoku Place, Hilo, HI 96720, USA

6 ESO, Karl-Schwarzschild Strasse 2, 85748 Garching-bei-München, Germany

7 Université Paris XI, Institut d'Astrophysique Spatiale, Bât. 121, 91450 Orsay Cedex, France

8 Max Plank Institut für Extraterrestrische Physik, Postfach 1603, 85740 Garching, Germany

Received 25 July 2000 / Accepted 13 November 2000

\begin{abstract}
We have observed an extended region surrounding the first discovered galactic superluminal source GRS 1915+105, seeking evidence of interaction between the relativistic ejecta of that object and the interstellar medium. We find two radio sources axisymmetrically aligned along the sub-arcsecond relativistic ejecta of GRS $1915+105$ and roughly $17^{\prime}$ distant from it, which coincide with the luminous IRAS sources $19124+1106$ and $19132+1035$. We have observed these sources at centimeter (VLA), millimeter (IRAM $30 \mathrm{~m}$ ), and infrared (ISO, UKIRT, ESO/MPI $2.2 \mathrm{~m}$ ) wavelengths in both line and continuum emission. At centimeter wavelengths a non-thermal jet-like feature aligned along the outflow axis is located adjacent to the inner edge of the southern source. Strong density enhancements are found in the millimeter tracers $\mathrm{CO}$ and $\mathrm{H}^{13} \mathrm{CO}^{+}$at the positions of both sources and some of the morphology is reminiscent of shock-like interactions; however, linewidths are narrow. At infrared wavelengths strong hydrogen recombination lines and weak lines of molecular hydrogen are observed at the southern source. We discuss these results as possible evidence of the sought-after interaction, both in terms of the regions undergoing ongoing shock-heating and in terms of them being locations of shock-induced star formation. The evidence for each of these is inconclusive. Millimeter line mapping of a portion of W 50 where the relativistic jets of the X-ray binary SS 433 interact with the interstellar medium shows roughly similar morphology as GRS 1915+105, suggesting that the phenomena observed at the IRAS sources may not be unusual for such a long distance interaction.
\end{abstract}

Key words. stars: individual: GRS 1915+105, SS 433 - ISM: individual objects: IRAS 19124+1106, IRAS 19132+1035 - ISM: jets and outflows - X-rays: stars

\section{Introduction}

The hard X-ray transient GRS 1915+105 was discovered in 1992 by the all-sky monitor WATCH on GRANAT (Castro-Tirado et al. 1994). It has been intensively studied since then in many wavebands, including the radio, where ejected plasma clouds with apparently superluminal velocities, a common extragalactic phenomenon, were found for the first time in our Galaxy (Mirabel \& Rodríguez 1994). Material ejected from GRS 1915+105 would be expected to interact with the surrounding interstellar medium, providing an opportunity to study in detail for the first time

Send offprint requests to: S. Chaty, e-mail: S.Chaty@open.ac.uk such a relativistic interaction (for a review see Mirabel \& Rodríguez 1999).

In a program to look for these interactions we have detected two compact sources of bright radio emission, each coincident with a bright IRAS source near GRS $1915+105$. These sources are located axisymetrically with respect to GRS $1915+105$ and at same position angle as its normal sub-arsec ejections, suggesting that they could be the zones of interaction between the ejecta and the interstellar medium as described in Sect. 2. We have observed these two IRAS sources at near-infrared (ESO/MPI $2.2 \mathrm{~m}$, UKIRT), mid-infrared (ISO), millimeter (IRAM $30 \mathrm{~m}$ ) and centimeter (VLA) wavelengths. The observations are detailed in Sect. 3. We discuss in 
Sect. 4 the possibility of a physical association between the ejections of GRS $1915+105$ and the two IRAS sources, comparing some of the results obtained in the putative interaction zones with new measurements of the interaction between one of the jets of SS 443 and the interstellar medium. Some of the observations and results described here have been briefly described in Chaty (1998), Chaty et al. (2000) and Rodríguez \& Mirabel (1998) (hereafter RM98).

\section{The context: Two axisymmetric sources around GRS $1915+105$}

In order to search for interactions involving the energetic and relativistic ejections of GRS 1915+105, the region surrounding GRS $1915+105$ was observed at radio wavelengths as described by RM98. This search was performed at $\lambda=20 \mathrm{~cm}$, using the Very Large Array (VLA) of $\mathrm{NRAO}^{1}$, in its C-configuration, giving a resolution of $15^{\prime \prime}$. The resulting map is shown in Fig. 1. The region in the map referred to as $\mathrm{G} 45.45+0.06$ was mislabelled G45.46+0.06 in the original papers (e.g., RM98). Characteristics of this radio source are given in Downes et al. (1980) and in Feldt et al. (1998). No evidence of jets or elongated clouds appears in the figure. However, two small radio continuum sources positioned nearly axisymmetrically with respect to GRS $1915+105$ were found at angular separations of $17^{\prime}$ each from GRS $1915+105$ (RM98). These small sources are coincident with the bright IRAS sources, 19124+1106 and 19132+1035. Their coordinates are given in Table 1 . The position angle of the line connecting the northwest source and GRS $1915+105$ is 157.9 , and their separation is $16^{\prime} .6$, the equivalent values for the southeastern source and GRS 1915+105 are 156.6 and $16^{\prime} .9$. The position angles are very similar to the position angle of the sub-arcsec radio-ejections from GRS 1915+105 ( 150) (Mirabel \& Rodríguez 1994; Fender et al. 1999). The angle between these ejections and the line of sight towards GRS $1915+105$ is $70^{\circ}$, with the southeastern ejection approaching and the northwestern one receding (Mirabel \& Rodríguez 1994).

The distance to GRS 1915+105 is crucial for determining many of the physical parameters of the object (e.g., mass loss rate, velocity of ejecta, energetics). It was estimated to be $12.5 \pm 1.5 \mathrm{kpc}$ by Mirabel \& Rodríguez (1994) and by Chaty et al. (1996). Recent VLBA observations are consistent with this value (Dhawan et al. 2000). However, the uncertainty may be larger than $1.5 \mathrm{kpc}$, as the above lower limit was based on a distance to G45.45+0.06 of $8.8 \mathrm{kpc}$, whereas Feldt et al. (1998) have placed the H II region at only $6.6 \mathrm{kpc}$. Moreover, Fender et al. (1999), who derived an upper limit of $11.2 \pm 0.8 \mathrm{kpc}$, consider that the source is constrained to lie between 7 and $12 \mathrm{kpc}$.

\footnotetext{
1 The National Radio Astronomy Observatory is operated by Associated Universities, Inc., under cooperative agreement with the USA National Science Foundation.
}

Table 1. Positions of GRS $1915+105$, IRAS $19124+1106$ and IRAS $19132+1035$. These coordinates are the positions of peak signal at $20 \mathrm{~cm}$ as observed by the VLA

\begin{tabular}{|c|l|l|}
\hline Source & J2000.0 coord. & gal. coord. \\
\hline GRS 1915+105 & $\alpha=19^{\mathrm{h}} 15^{\mathrm{m}} 11^{\mathrm{s}} .545$ & $l^{\mathrm{II}}=45^{\circ} .40$ \\
& $\delta=10^{\circ} 56^{\prime} 44^{\prime \prime} .80$ & $b^{\mathrm{II}}=-0.29$ \\
\hline IRAS 19124+1106 & $\alpha=19^{\mathrm{h}} 14^{\mathrm{m}} 45^{\mathrm{s}} .77$ & $l^{\mathrm{II}}=45^{\circ} .54$ \\
& $\delta=11^{\circ} 12^{\prime} 06^{\prime \prime} .4$ & $b^{\mathrm{II}}=-0.007$ \\
\hline IRAS 19132+1035 & $\alpha=19^{\mathrm{h}} 15^{\mathrm{m}} 39^{\mathrm{s}} 13$ & $l^{\mathrm{II}}=45^{\circ} .19$ \\
& $\delta=10^{\circ} 41^{\prime} 17^{\prime \prime} \cdot 1$ & $b^{\mathrm{II}}=-0.44$ \\
\hline
\end{tabular}

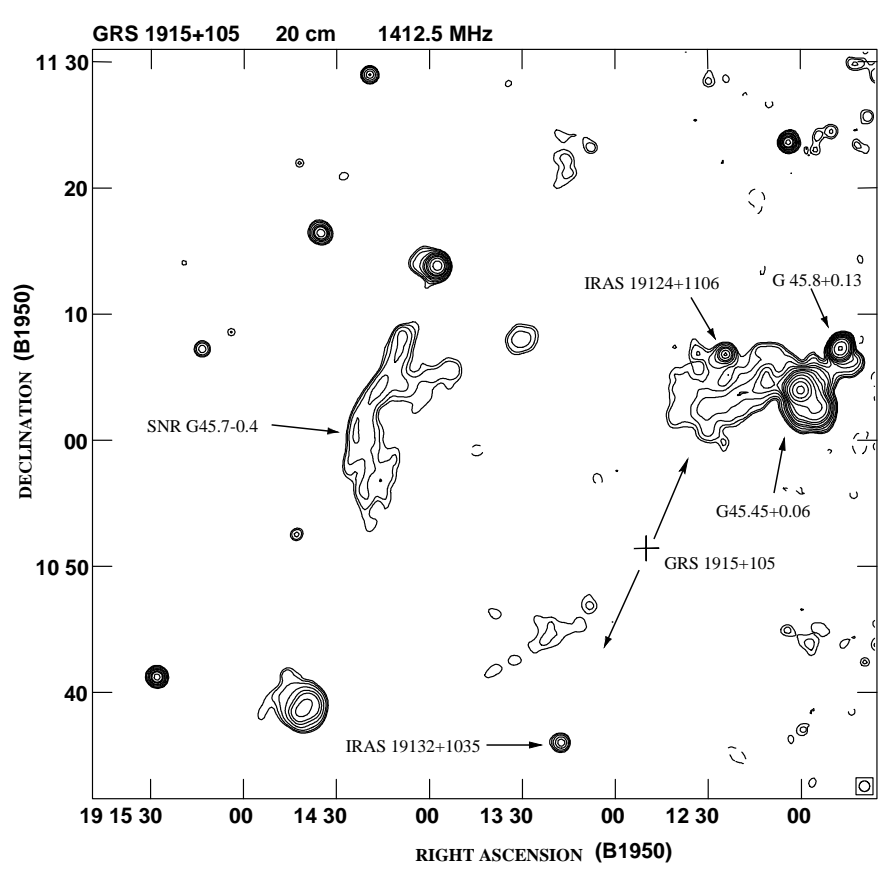

Fig. 1. Map of the surroundings of GRS $1915+105$, taken with the VLA-C at $\lambda=20 \mathrm{~cm}$. Contour levels are $-3,3,4,6,10,15$, $20,30,40,60,100,200,400$ and $800 \times 3$ mJy beam $^{-1}$. The half power contour of the beam is shown in the bottom right corner. The arrows around GRS 1915+105 indicate the position angle of the sub-arc relativistic ejecta

If the radio sources are at the same distances from the Sun as GRS $1915+105$, and if we assume that the distance of this source is $12.5 \pm 1.5 \mathrm{kpc}$, their angular separations correspond to the distance of 60 pc from GRS $1915+105$.

\section{Observations and results}

\subsection{Centimeter}

High-resolution maps of the two radio sources have been obtained with the VLA. Details of these observations can be found in RM98. These maps, at three different wavelengths, 2, 6 and $20 \mathrm{~cm}$, are shown in Fig. 2. The morphology of the northern source resembles that of a cometary 
Table 2. Flux densities of IRAS $19124+1106$ and IRAS $19132+1035$. All the flux densities with $\lambda$ from $12 \mu \mathrm{m}$ to $20 \mathrm{~cm}$ come from RM98. We refer to Table 3 for the fluxes at mid-infrared wavelengths

\begin{tabular}{|llllcccccc|}
\hline Source & $1.25 \mu \mathrm{m}$ & $2.2 \mu \mathrm{m}$ & $12 \mu \mathrm{m}$ & $25 \mu \mathrm{m}$ & $60 \mu \mathrm{m}$ & $100 \mu \mathrm{m}$ & $2 \mathrm{~cm}$ & $6 \mathrm{~cm}$ & $20 \mathrm{~cm}$ \\
& $\mathrm{mag}$ & $\mathrm{mag}$ & $\mathrm{Jy}$ & $\mathrm{Jy}$ & $\mathrm{Jy}$ & $\mathrm{Jy}$ & $\mathrm{mJy}$ & $\mathrm{mJy}$ & $\mathrm{mJy}$ \\
\hline IRAS 19124+1106 & $17.9 \pm 0.1$ & $12.1 \pm 0.1$ & 3.9 & 19.6 & 260.6 & 581.5 & $114 \pm 6$ & $130 \pm 4$ & $114 \pm 4$ \\
\hline IRAS 19132+1035 & $17.45 \pm 0.1$ & $10.7 \pm 0.1$ & 6.9 & 34.0 & 277.4 & 488.8 & $52 \pm 6$ & $63 \pm 4$ & $60 \pm 4$ \\
\hline IRAS 19132+1035 "jet" & & & & & & & $\leq 1$ & 2 & 5 \\
\hline
\end{tabular}

HII region, but it also shows a bow shock-like structure to the South-East, e.g. towards GRS 1915+105. At the southern lobe a non-thermal jet extends to the northwest along the line between the source and GRS 1915+105. The flux densities of this jet are $\lesssim 1,2$ and 5 mJy at 2,6 and $20 \mathrm{~cm}$, respectively, indicating a spectral index of $\alpha=-0.8\left(f_{\nu} \propto \nu^{\alpha}\right)$, therefore noticeably different than that of the rest of the southern radio lobe, which exhibits thermal emission, as it can be seen in the Table 2. We also reported in Fig. $3^{2}$ a spectral index map of IRAS $19132+1035$ made from the 20 and 6 -cm maps. The southern lobe also shows a sharp edge to the south, which could be either a bow shock, or the ionization front of an $\mathrm{H}$ II region.

Additional radio observations of these sources in the H92 $\alpha$ recombination line and adjacent continuum at $3.6 \mathrm{~cm}$ are detailed in RM98. The line strengths, profiles, and radial velocities are typical of $\mathrm{H}$ II regions at kinematic distances of $7.4 \pm 1.4 \mathrm{kpc}$ for the north lobe and $6.0 \pm 1.4 \mathrm{kpc}$ for the south lobe (distance errors are estimated from the mean value for deviations of circular rotation of $12 \mathrm{~km} \mathrm{~s}^{-1}$ given by Brand \& Blitz 1993). We note that the distance uncertainties are large because of the low velocity resolution $\left( \pm 12 \mathrm{~km} \mathrm{~s}^{-1}\right)$ of the H92 observations (RM98). These estimates are most consistent with the distance to G45.45+0.06 given by Feldt et al. (1998). The radio luminosities of the sources derived using these distances are similar to those of H II regions powered by O9.5 ZAMS and B0 ZAMS stars (RM98). If instead they are at the nominal distance of GRS $1915+105$ hotter individual stars or two stars of the above spectral types would be required to power each source. However, the interpretation of these objects as normal HiI regions does not explain the presence of the non-thermal jet-like structure in IRAS 19132+1035. Furthermore, given the large uncertainty in the distance of GRS $1915+105$, we cannot a priori rule out the possibility that GRS $1915+105$ is roughly at the kinematic distance of the $\mathrm{H}$ II regions.

A search for $\mathrm{OH}$ maser emission was performed with the VLA in the vicinity of GRS $1915+105$, at $1720 \mathrm{MHz}$. Such emission can be a signpost for interaction between a SNR and molecular gas (as in the case of IC 443, e.g. Denoyer 1979). No detection was obtained. However, this does not imply necessarily that there is no interaction (Rodríguez, Goss, Mirabel, private communication).

\footnotetext{
${ }^{2}$ On the color page at the end of this article.
}

\subsection{Infrared}

\subsubsection{Near-infrared}

Broad-band imaging ${ }^{3}$

We imaged the IRAS sources in the $J(1.25 \mu \mathrm{m})$ and $K$ $(2.2 \mu \mathrm{m})$ bands. The observations were made on 1997, April 5, with IRAC2b installed on the Max Planck Institute's $2.2 \mathrm{~m}$ telescope at the European Southern Observatory (ESO). The IRAC2b camera, which contains a Rockwell $256 \times 256$ pixel Hg:Cd:Te NICMOS 3 array detector was mounted at the $\mathrm{f} / 35$ infrared adapter of the telescope. It was used with lens $\mathrm{C}$, providing an image scale of $0.49 \mathrm{arcsec} / \mathrm{pixel}$ and a field of $136 \times 136 \mathrm{arcsec}^{2}$. The typical seeing for these observations was 1.2 arcsec. Each final image is the median of 5 frames, each exposed for 2 min. After taking each image of the object, an image of adjacent sky was taken, to allow subtraction of sky emission. The images were processed by removing bias and dark current, and applying a flat field correction. These steps were performed using IRAF procedures, in particular the DAOPHOT package for the photometry in crowded fields.

The images reveal a compact near-infrared counterpart at the position of IRAS $19124+1106$, with $J=$ $17.9 \pm 0.1 \mathrm{mag}$ and $K=12.1 \pm 0.1 \mathrm{mag}$, and a brighter and more extended counterpart of IRAS $19132+1035$, with $J=17.45 \pm 0.1 \mathrm{mag}$ and $K=10.7 \pm 0.1 \mathrm{mag}$. Figure 4 shows the central portions of the images containing IRAS $19132+1035$.

\section{Spectroscopy}

A low resolution $K$-band spectrum of IRAS $19132+1035$ was obtained with the facility instrument CGS4 on the UK Infrared $3.8 \mathrm{~m}$ Telescope (UKIRT) on UT 1997 July 13, as part of the UKIRT Service Program. The $75 \mathrm{l} / \mathrm{mm}$ grating was used in CGS4 together with a slit of width $1.2^{\prime \prime}$ to provide a spectral coverage of $0.67 \mu \mathrm{m}$ at a resolution of $0.0026 \mu \mathrm{m}\left(370 \mathrm{~km} \mathrm{~s}^{-1}\right.$ at $\left.2.15 \mu \mathrm{m}\right)$ on the $256 \times 256$ array of InSb detectors. The slit was oriented at a position angle of $148^{\circ}$ and the telescope was moved to center the peak signal from the infrared counterpart in one row of the array. Observations were obtained in the standard

\footnotetext{
${ }^{3}$ Based on observations collected at the European Southern Observatory, Chile.
} 

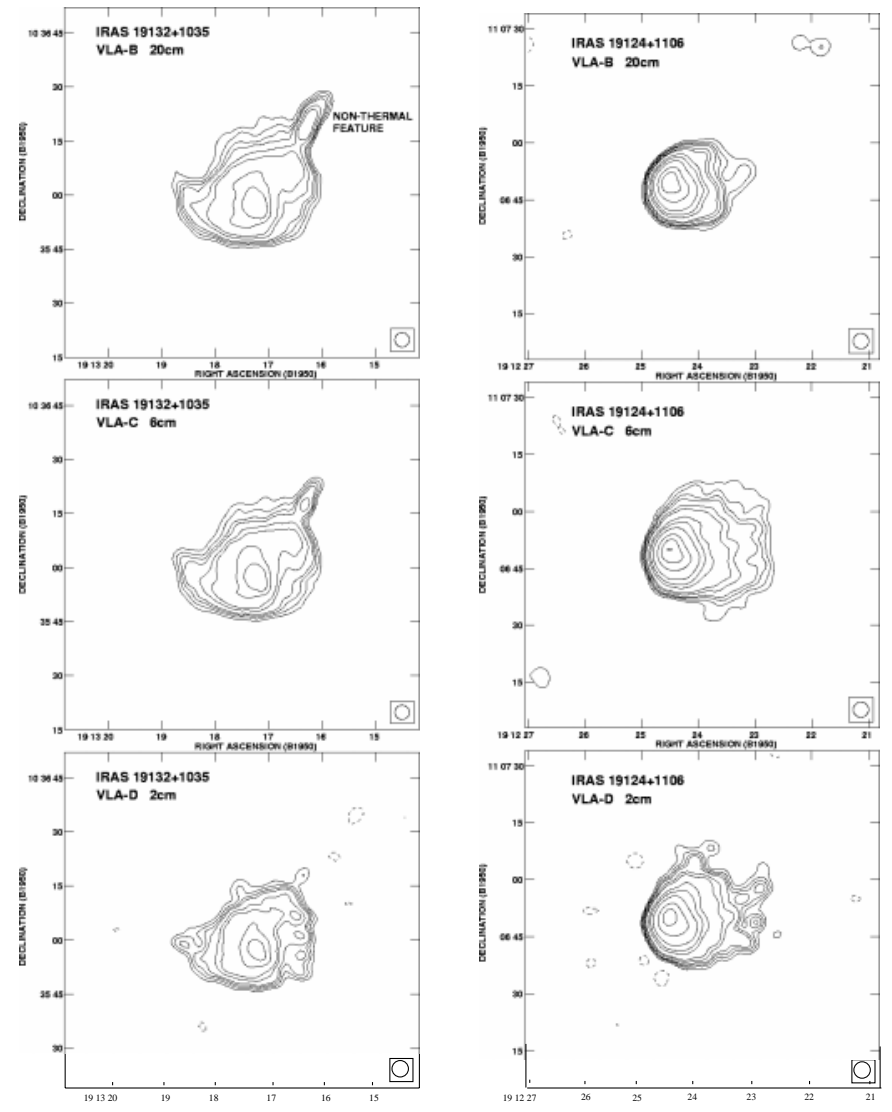

Fig. 2. Maps of the two continuum radio sources IRAS 19132+1035 (left) and IRAS 19124+1106 (right), acquired with the VLA respectively in the D-configuration for the $\lambda=2 \mathrm{~cm}$ map (bottom), in the C-configuration for the $\lambda=6 \mathrm{~cm}$ map (middle) and in the B-configuration for the $\lambda=20 \mathrm{~cm}$ map (top). Contour levels are $-4,4,6,8,10,15,20,40,60,100,200,300$ and $400 \times$ the $\mathrm{rms}$ noise of $0.05 \mathrm{mJy}^{-1}$ beam ${ }^{-1}$ The half power contour of the beam, with diameter of $4^{\prime \prime}$, is shown in each bottom right corner

stare/nod-along-slit mode. The total integration time was 8 minutes. A near simultaneous spectrum of the F3V star HR 6987 ( $T=6700 \mathrm{~K}, K=4.50$ assumed) was obtained at the same airmass; in order to remove telluric absorption lines (the Brackett $\gamma(7-4)$ absorption line, with a central depth of 0.88 of the continuum at this spectral resolution, was artificially removed from the spectrum of the comparison star prior to ratioing). Wavelength calibration was obtained from observations of an argon arc lamp and is accurate to $0.0005 \mu \mathrm{m}$.

The spectrum of IRAS $19132+1035$ is shown in Fig. 5 . It is the sum of the spectra of three adjacent rows, covering an area of $1.2 \times 3.6$ arcsec. Most of the flux is contained in the central row. The spectrum exhibits a very red continuum and a number of emission lines. Most prominent are three recombination lines of atomic hydrogen: a very strong Paschen $\alpha(4-3)$ line at $1.876 \mu \mathrm{m}$, and weaker Brackett $\gamma(7-4)$ and Brackett $\delta(8-4)$ lines, at $2.166 \mu \mathrm{m}$
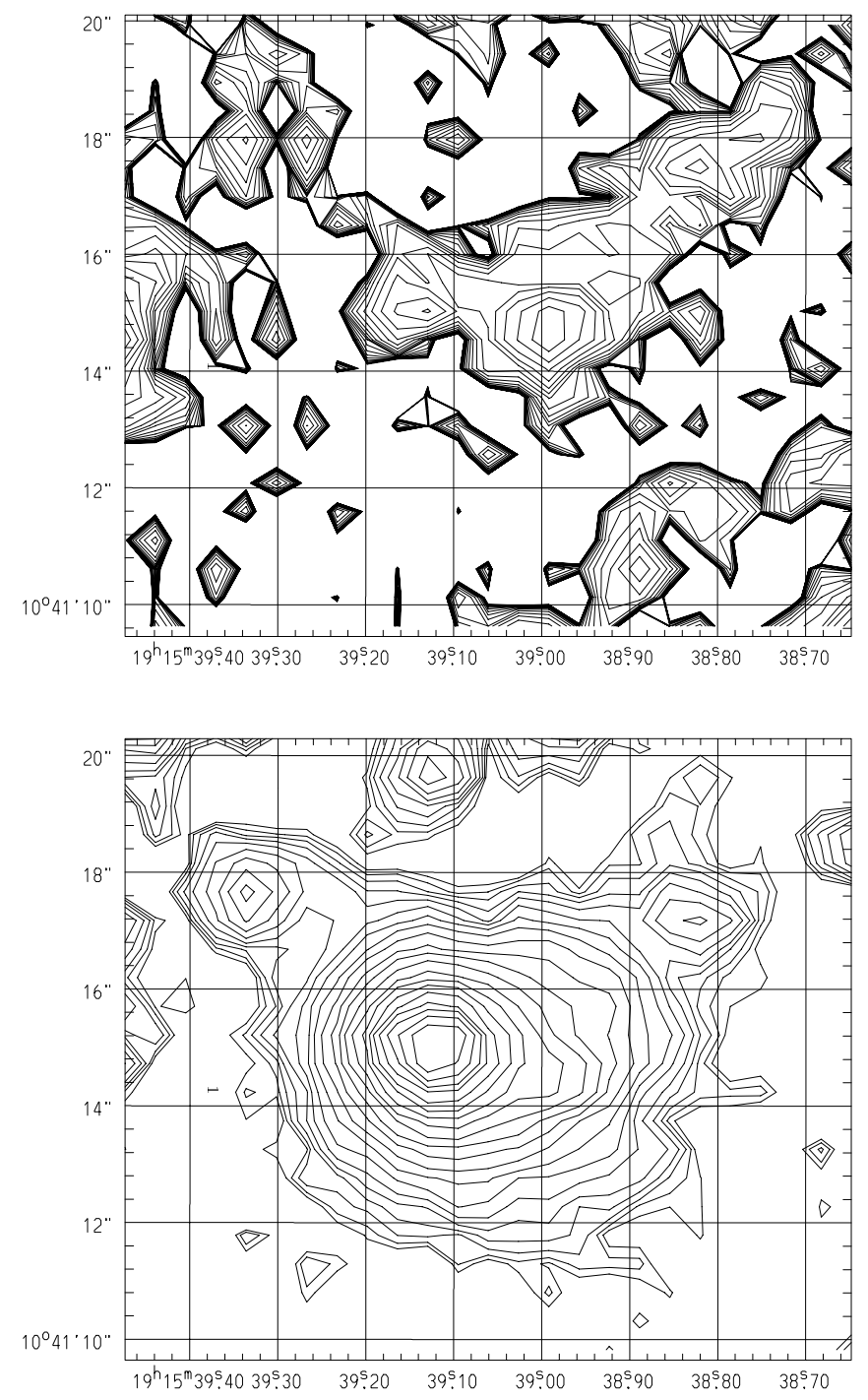

Fig. 4. $J$ (top) and $K$ (bottom) band images of the source IRAS $19132+1035$, taken with IRAC $2 \mathrm{~b}$ on the $2.2 \mathrm{~m}$ of the ESO. The coordinates are for the J2000 equinox. The ADU flux contour levels are in a logarithmic scale, each separated by a factor $\sqrt{2}$, the first one is equal respectively to 0.864 for the $J$ band and to 25.501 for the $K$ band

and $1.945 \mu \mathrm{m}$, respectively. The $\operatorname{Pa} \alpha$ and $\operatorname{Br} \delta$ lines are uncorrected for the absorption due to the same line in the comparison star and both it and the $\mathrm{Br} \delta$ line occur near strong telluric absorption lines of water vapor; thus their strengths and profiles are subject to systematic errors.

The peak of the $\operatorname{Br} \gamma$ emission line occurs at $+45 \pm 70 \mathrm{~km} \mathrm{~s}^{-1}$ in the Local Standard of Rest (LSR). The Full Width at Half Maximum (FWHM) of the profile is only marginally broader than the instrumental resolution, but the profile appears to exhibit a weak highvelocity wing, displaced towards the red. No such wing is present in the profile of either of the other two atomic hydrogen lines. We note that lines displaced towards the blue are much more common in the $\mathrm{H}$ II regions, because the front part of an expanding $\mathrm{H}$ II region is more easily detected. 


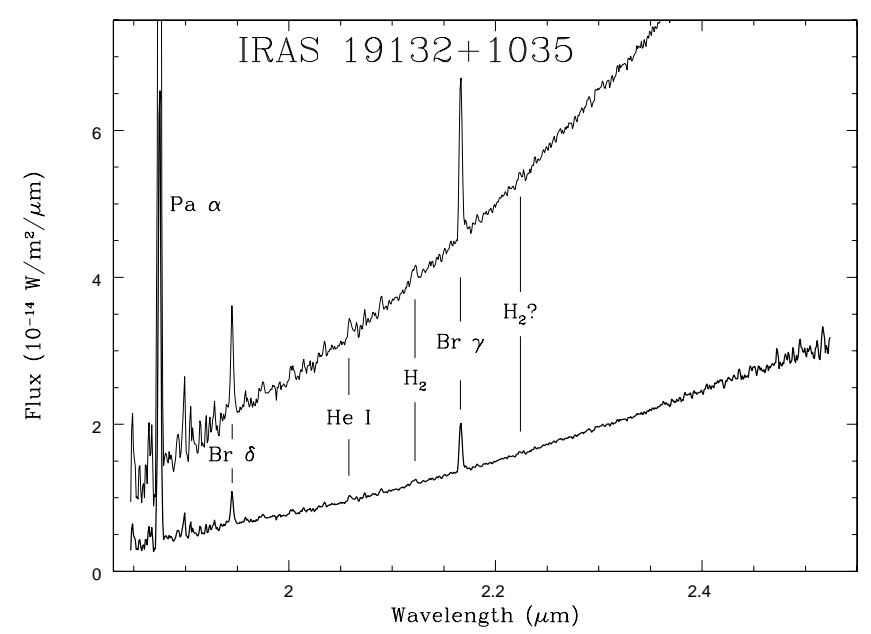

Fig. 5. $K$-band spectrum of IRAS $19132+1035$ obtained with CGS 4 on UKIRT. The upper trace is multiplied by a factor of 3.33 to allow an easier reading of the weakest lines. Identifications and positions of detected and marginal lines are indicated

Two fainter emission lines also are visible: the singlet He I $2 \mathrm{P}-2 \mathrm{~S}$ transition at $2.059 \mu \mathrm{m}$ and the $\mathrm{H}_{2} 1-0 S(1)$ line at $2.122 \mu \mathrm{m}$. The flux in the $\mathrm{H}_{2}$ line is $\sim 10^{-17} \mathrm{~W} \mathrm{~m}^{-2}$. There is also evidence for the presence of the $\mathrm{H}_{2} 2-1 S(1)$ line at $2.224 \mu \mathrm{m}$, at roughly one-fourth the strength of the 1-0 line, which suggests that most of the excitation of $\mathrm{H}_{2}$ is due to absorbed UV radiation rather than collisions. The 1-0 line appears partly resolved with a deconvolved FWHM of $\sim 500 \mathrm{kms}^{-1}$, but new measurements are required to confirm this. The signal-to-noise ratio of the $2-1$ line is too low to determine if its profile is resolved.

\subsubsection{Mid-infrared}

Both infrared sources were imaged in six wavebands from 7 to $15 \mu \mathrm{m}$ with the infrared camera ISOCAM ${ }^{4}$ (Cesarsky et al. 1996), on the Infrared Space Observatory (ISO) satellite mission as part of the Guaranteed and Open Time Programme. The observations were performed on 1996 April 28 and on 1997 October 20. The log of the observations and the derived fluxes in all bands are found in Table 3. The images were taken through the Large Width (LW) filters at angular resolutions given in this Table. Data reduction used the CIA package and included subtraction of the dark current, suppression of the cosmic ray impacts by a multiresolution median method, correction of the detector transient behaviour, flat field correction and when necessary a distortion correction (see Starck et al. 1999 and references therein).

The observation at $7 \mu \mathrm{m}$ of the south lobe, shown at the bottom of Fig. $6^{5}$, is particularly striking, because

\footnotetext{
${ }^{4}$ ISOCAM was constructed under the scientific direction and the technical expertise of the Service d'Astrophysique of the CEA/Saclay. The ISOCAM Consortium is led by the PI C. Cesarsky.

${ }^{5}$ On the color page at the end of this article.
}

it shows thermal emission, presumably from heated dust, exactly coincident with the radio counterpart. One component of this emission is localized, between the maximum and the jet feature seen at radio wavelengths, on a line connecting GRS 1915+105 and IRAS 19132+1035. The other component is more extended, and closely resembles the remainder of the radio image with its bow-shock morphology at the south-east edge. The $15 \mu \mathrm{m}$ image of the north lobe is shown at the top of Fig. 6. The morphology is similar to the radio images, resembling a common cometary H II region, but with two bow shock-like structures facing each other.

\subsection{Millimeter}

We used the $30 \mathrm{~m}$ radio telescope of the Instituto de Radio Astronomía Milimetrica $\left(\mathrm{IRAM}^{6}\right)$ to observe molecular lines which are good density tracers and are diagnostics of shock excitation and chemistry.

The observations, totalling 70 hours, took place during 1997 April 17-24, November 29-December 3, and during 1997 December 31-1998 January 5. The observed molecules and transitions, together with their respective frequencies were: ${ }^{12} \mathrm{CO}(J=2-1)$ at $\nu=230.5 \mathrm{GHz}$, ${ }^{13} \mathrm{CO}(J=2-1)$ at $\nu=220.4 \mathrm{GHz}, \mathrm{H}^{13} \mathrm{CO}^{+}(J=1-0)$ at $\nu=89.2 \mathrm{GHz}, \mathrm{SiO}(J=2-1, v=0)$ at $\nu=86.8 \mathrm{GHz}$, $\mathrm{SiO}(J=3-2, v=0)$ at $\nu=130.3 \mathrm{GHz}, \mathrm{SiO}(J=$ $5-4, v=0)$ at $\nu=217.1 \mathrm{GHz}$ and $\mathrm{CS}(J=2-1)$ at $\nu=98.0 \mathrm{GHz}$. Details are given in Table 4 .

We used position switching during the observations, with the off-source position located $\left(-500^{\prime \prime},-1200^{\prime \prime}\right)$ from the position of GRS $1915+105$. Tests demonstrated that the off position contained no significant line emission. The treatment and analysis of the millimetric data were standard, consisting for each spectrum of a subtraction of a polynomial baseline of first or second order, in order to remove instrumental fringes.

\subsubsection{IRAS $19132+1035$}

For each of the ${ }^{12} \mathrm{CO},{ }^{13} \mathrm{CO}, \mathrm{H}^{13} \mathrm{CO}^{+}$and $\mathrm{SiO} 2-1$ transitions, we obtained 23 spectra at positions separated by $6^{\prime \prime}$, along the line joining GRS $1915+105$ to the South lobe and crossing the maximum of the centimetric continuum shown at the bottom left panel of Fig. 2; this maximum was defined as the $(0,0)$ position. Integration times were 15 min per point, except at the $(0,0)$ position where it was $45 \mathrm{~min},(-13,20)$ where it was $135 \mathrm{~min}$, and $(-10,15)$ where it was $90 \mathrm{~min}$. The two latter locations correspond to the jet-like feature. In addition we obtained spectra at eight locations adjacent to the jet. The observations of $\mathrm{CS}, \mathrm{SiO} 3-2$ and $\mathrm{SiO}$ 5-4 each

6 IRAM is an European institute for millimeter astronomy, founded by the French Centre National de la Recherche Scientifique (CNRS) and the German Max-Planck-Gesellschaft (MPG), in collaboration with the Spanish Instituto Geografico Nacional (IGN). 
Table 3. Log, characteristics and fluxes of the sources from ISO observations. PFOV $=$ Pixel Field Of View

\begin{tabular}{c|c|c|c|c}
\hline Source & Date & Filter & PFOV & Flux \\
\hline IRAS 19132+1035 & $28 / 04 / 96$ & LW2 $(5-8.5 \mu \mathrm{m})$ & $3^{\prime \prime}$ & $13.2 \pm 2.6 \mathrm{Jy}$ \\
IRAS 19132+1035 & $28 / 04 / 96$ & LW7 $(8.7-10.7 \mu \mathrm{m})$ & $6^{\prime \prime}$ & $5.9 \pm 1.2 \mathrm{Jy}$ \\
IRAS 19132+1035 & $28 / 04 / 96$ & LW3 $(12-18 \mu \mathrm{m})$ & $6^{\prime \prime}$ & $10.7 \pm 2.2 \mathrm{Jy}$ \\
IRAS 19132+1035 & $20 / 10 / 97$ & LW10 $(8-15 \mu \mathrm{m})$ & $1.5^{\prime \prime}$ & $6.3 \pm 1.2 \mathrm{Jy}$ \\
IRAS 19124+1106 & $20 / 10 / 97$ & LW9 $(14-16 \mu \mathrm{m})$ & $3^{\prime \prime}$ & $3.3 \pm 0.66 \mathrm{Jy}$ \\
\hline
\end{tabular}

Table 4. Frequencies of the transitions and receivers used at IRAM. The filter bank b3*, divided into sections b31, b32, b33 and b34, provides a low resolution of $1 \mathrm{MHz}$, and contains $4 \times 256$ channels, for a total bandwidth of $1 \mathrm{GHz}$; the filterbank b20 provides a high resolution of $100 \mathrm{kHz}$, and contains $2 \times 128$ channels, for a total bandwidth of $25.3 \mathrm{MHz}$; the autocorrelator b4*, divided into sections b41, b42, b43 and b44 offers resolutions ranging from $10 \mathrm{kHz}$ to $1.25 \mathrm{MHz}$, a variable number of channels, and a variable total bandwidth from $20 \mathrm{MHz}$ to $1 \mathrm{GHz}$

\begin{tabular}{llllll}
\hline Abbr & Transition & Frequency & Receiver & Banks & Beam \\
\hline${ }^{12} \mathrm{CO}$ & ${ }^{12} \mathrm{CO}(J=2-1)$ & $\nu=230.537990 \mathrm{GHz}$ & $230 \mathrm{~g} 2$ & $\mathrm{~b} 44 \mathrm{~b} 34$ & $10.9^{\prime \prime}$ \\
${ }^{13} \mathrm{CO}$ & ${ }^{13} \mathrm{CO}(J=2-1)$ & $\nu=220.398686 \mathrm{GHz}$ & $230 \mathrm{~g} 1$ & $\mathrm{~b} 43 \mathrm{~b} 33 \mathrm{~b} 20$ & $11.4^{\prime \prime}$ \\
$\mathrm{H}^{13} \mathrm{CO}^{+}$ & $\mathrm{H}^{13} \mathrm{CO}^{+}(J=1-0)$ & $\nu=89.188523 \mathrm{GHz}$ & $3 \mathrm{~mm} 1$ & $\mathrm{~b} 42 \mathrm{~b} 32$ & $28.2^{\prime \prime}$ \\
$\mathrm{CS}$ & $\mathrm{CS}(J=2-1)$ & $\nu=97.980968 \mathrm{GHz}$ & $3 \mathrm{~mm} 1$ & $\mathrm{~b} 41 \mathrm{~b} 31$ & $25.7^{\prime \prime}$ \\
$\mathrm{SiO} \mathrm{2-1}$ & $\mathrm{SiO}(J=2-1, v=0)$ & $\nu=86.846891 \mathrm{GHz}$ & $3 \mathrm{~mm} 2$ & $\mathrm{~b} 41 \mathrm{~b} 31$ & $29^{\prime \prime}$ \\
$\mathrm{SiO} \mathrm{3-2}$ & $\mathrm{SiO}(J=3-2, v=0)$ & $\nu=130.268702 \mathrm{GHz}$ & $2 \mathrm{~mm}$ & $\mathrm{~b} 42 \mathrm{~b} 32$ & $19.4^{\prime \prime}$ \\
$\mathrm{SiO} \mathrm{5-4}$ & $\mathrm{SiO}(J=5-4, v=0)$ & $\nu=217.104935 \mathrm{GHz}$ & $230 \mathrm{~g} 1$ & $\mathrm{~b} 43 \mathrm{~b} 33 \mathrm{~b} 20$ & $11.6^{\prime \prime}$ \\
\hline
\end{tabular}

consisted of 15 spectra at $6^{\prime \prime}$ separation along the same line, with integration times of 15 min per position with the exception of $(0,0)$ and $(10,-15)$ where the times were 30 and $7 \mathrm{~min}$, respectively.

Results for ${ }^{12} \mathrm{CO},{ }^{13} \mathrm{CO}, \mathrm{H}^{13} \mathrm{CO}^{+}$and $\mathrm{CS}$

Figure $7^{7}$ contains position-velocity plots with an expanded velocity scale for the observed lines of ${ }^{12} \mathrm{CO},{ }^{13} \mathrm{CO}$, $\mathrm{H}^{13} \mathrm{CO}^{+}$and $\mathrm{CS}$.

For all transitions the line peak occurs at an LSR velocity of $67 \mathrm{~km} \mathrm{~s}^{-1}$, close to the velocity of $75.7 \mathrm{~km} \mathrm{~s}^{-1}$ of the H92 $\alpha$ recombination line observations as seen by RM98 with much lower velocity resolution. The intensity distributions of the millimeter lines are not symmetric, each showing a sharper edge to the south (positive RA offset), as seen in the VLA images, and a slightly shifted peak velocity. The CS transition, which is a high density tracer, exhibits a peak shifted to the northwest (more negative RA offsets), towards the jet-like feature.

\section{$\mathrm{SiO}$}

The $\mathrm{SiO} 2-1$ transition was detected only close to the position $(-10,14)$; the spectrum at that location is shown in Fig. 8. The line is weak, but its velocity, $67.6 \pm 0.5 \mathrm{kms}^{-1}$ and width, $4.4 \pm 1.2 \mathrm{kms}^{-1}$ are similar to those seen for much stronger lines and give us confidence that it is real. The 3-2 transition was not detected at any individual position, but the average of the spectra between $(0,0)$ and $(-17,25)$ yields a $3 \sigma$ detection

\footnotetext{
7 On the color page at the end of this article.
}

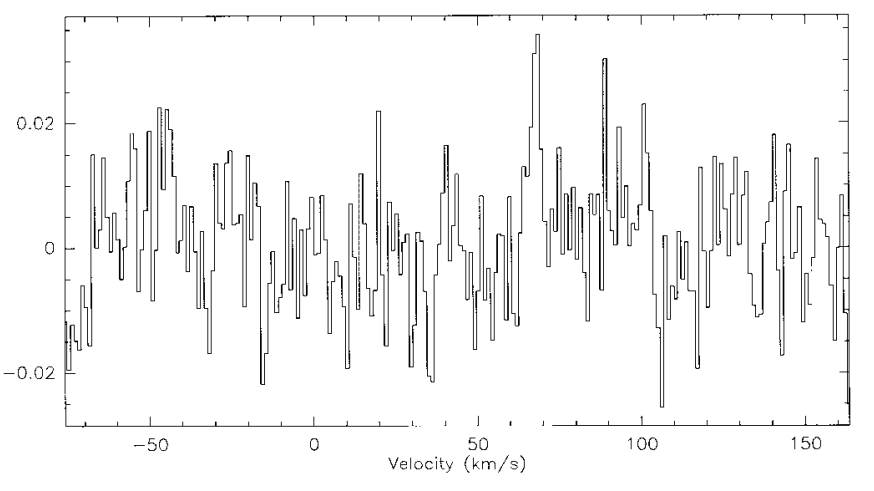

Fig. 8. Spectrum of the $\mathrm{SiO} 2-1$ line at IRAS $19132+1035$, obtained at the offset $(\alpha, \delta)=\left(-10^{\prime \prime},+14^{\prime \prime}\right)$. In this and the following spectra, the $y$-axis is the antenna temperature in $K$ and the $x$-axis is the radial velocity with respect to the LSR

of a narrow $\left(1.0 \pm 0.3 \mathrm{~km} \mathrm{~s}^{-1}\right)$ line at $67.3 \mathrm{~km} \mathrm{~s}^{-1} \pm 0.2$, exhibiting an antenna temperature of $T_{A}^{*}=0.044 \mathrm{~K}$ and an intensity of $0.05 \pm 0.01 \mathrm{~K} \mathrm{~km} \mathrm{~s}^{-1}$ (Fig. 9). The $\mathrm{SiO} 5-4$ transition was not detected at any individual location or when all of the spectra were averaged.

\section{Discussion}

Figure 7 demonstrates clearly that brightness maxima for transitions with higher critical densities are displaced increasingly towards the northwest where the non-thermal jet-like structure is located. What little $\mathrm{SiO}$ emission is detected also originates largely in this region. The very densest region could be the main interaction zone between the putative jet and the ambient medium. However, the narrow lines that are observed are difficult to explain in 


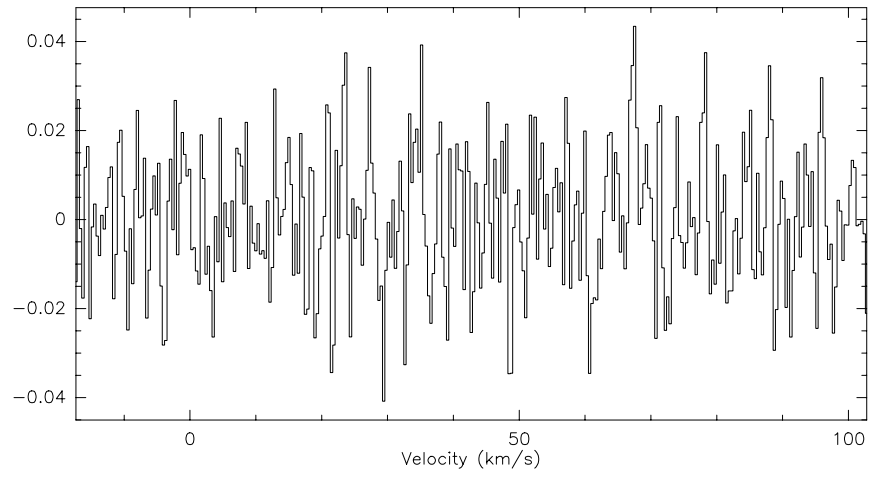

Fig. 9. Spectrum of the SiO $3-2$ line at IRAS $19132+1035$, averaged over all positions from $(0,0)$ to $(-17,25)$

the above scenario, as an energetic shock might be expected to lead to line emission over a much wider range of velocities than is observed. If a shock is present, it apparently must be of very low velocity.

\subsubsection{IRAS $19124+1106$}

For the ${ }^{12} \mathrm{CO},{ }^{13} \mathrm{CO}, \mathrm{H}^{13} \mathrm{CO}^{+}$and $\mathrm{SiO} 2-1$ transitions, we acquired 24 spectra at positions separated by $6^{\prime \prime}$, along the line joining GRS $1915+105$ to the northern lobe. As in the case of the southern lobe, the reference position $(0,0)$ is defined as the position of maximum radio continuum signal in the northern lobe, as observed by the VLA. The integration time at each position was 5 min, except at the reference position, where it was $10 \mathrm{~min}$. Each of the above transitions was detected over a wide range of positions. Spectra of the CS 2-1, $\mathrm{SiO} 3-2$ and $\mathrm{SiO} 5-4$ transitions were obtained only at the $(0,0)$ position, each with an integration time of $15 \mathrm{~min}$. None of the three $\mathrm{SiO}$ transitions were detected at any position at an upper limit of $0.05 \mathrm{~K}$, or when all positions were averaged.

Results for ${ }^{12} \mathrm{CO},{ }^{13} \mathrm{CO}$ and $\mathrm{H}^{13} \mathrm{CO}^{+}$

Position velocity diagrams for ${ }^{12} \mathrm{CO},{ }^{13} \mathrm{CO}$ and $\mathrm{H}^{13} \mathrm{CO}^{+}$ are presented using an expanded velocity scale in Fig. $10^{8}$. Two strong velocity components are readily apparent in all three transitions. For ${ }^{12} \mathrm{CO}$ at the $(0,0)$ position, the strongest component is centered at $53.95 \pm 0.03 \mathrm{~km} \mathrm{~s}^{-1}$, with a width of $4.05 \pm 0.09 \mathrm{~km} \mathrm{~s}^{-1}$, an intensity of $12.9 \mathrm{~K}$, and an integrated brightness of $55.5 \pm 0.09 \mathrm{~K} \mathrm{~km} \mathrm{~s}^{-1}$. For ${ }^{13} \mathrm{CO}$, this component is peaked at $55.10 \pm 0.04 \mathrm{kms}^{-1}$, and has a width of $3.88 \pm 0.10 \mathrm{~km} \mathrm{~s}^{-1}$, an intensity of $7.32 \mathrm{~K}$, and an integrated brightness of $30.3 \pm 0.60 \mathrm{~K} \mathrm{~km} \mathrm{~s}^{-1}$. A second velocity component occurs at $59.5 \pm 0.1 \mathrm{~km} \mathrm{~s}^{-1}$ with a width of $3.9 \pm 0.2 \mathrm{~km} \mathrm{~s}^{-1}$, an intensity of $3.11 \mathrm{~K}$ and an integrated brightness of $12.8 \pm 0.6 \mathrm{~K} \mathrm{~km} \mathrm{~s}^{-1}$ at the ${ }^{12} \mathrm{CO}$ $(0,0)$ position. The velocities of these two components are close to that of the H92 $\alpha$ recombination line peak at $57.3 \mathrm{~km} \mathrm{~s}^{-1}$ (RM98). A third velocity component, at

\footnotetext{
${ }^{8}$ On the color page at the end of this article.
}

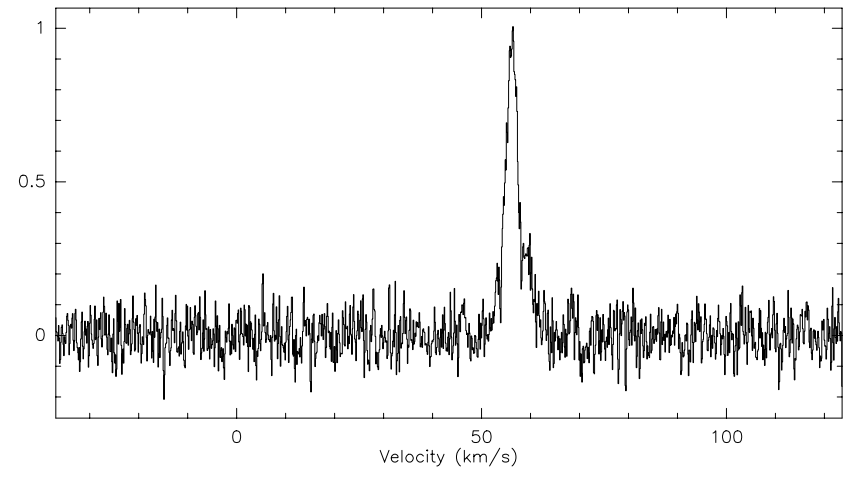

Fig. 11. IRAS $19124+1106$, CS, b41, spectrum taken at the position $(0,0)$

$\sim 6 \mathrm{kms}^{-1}$ is visible in the northwestern part of this cloud for $\mathrm{CO}$ and in the southeastern part for $\mathrm{H}^{13} \mathrm{CO}^{+}$. Given the very different velocity the line emission probably originates in a foreground or background cloud.

\section{Results for $C S$}

The single spectrum obtained at $(0,0)$, shown in Fig. 11, contains a strong component centered at $56.17 \pm 0.03 \mathrm{~km} \mathrm{~s}^{-1}$, of width $3.039 \pm 0.07 \mathrm{~km} \mathrm{~s}^{-1}$ intensity $0.937 \mathrm{~K}$ and integrated brightness $3.03 \pm$ $0.06 \mathrm{~K} \mathrm{~km} \mathrm{~s}^{-1}$. Adjacent to it is a weaker component at $59.91 \pm 0.11 \mathrm{kms}^{-1}$, with width $1.589 \pm 0.22 \mathrm{kms}^{-1}$, intensity $0.298 \mathrm{~K}$ and integrated brightness $0.504 \pm$ $0.07 \mathrm{~K} \mathrm{~km} \mathrm{~s}^{-1}$. These velocities are similar to those detected in other molecules and in H92 $\alpha$ (RM98).

\section{Discussion}

As already noted by RM98 from the centimeter observations, the morphology of the northern source is that of a cometary H II region. The millimeter wave spectroscopy, which reveals two velocity components, is consistent with this morphology.

\section{Discussion}

The new data do not definitively prove or disprove an association between GRS $1915+105$ and either of the IRAS sources. The strongest lines of evidence supporting an association are (1) the axisymmetric locations of the two sources at nearly the same position angle as the recent sub-arcsecond ejections observed at radio wavelengths, (2) the spatial coincidence of the non-thermal radio jet with the inner edge of the southern source, IRAS 19132+1035, as well as the orientation of this jet along the axis, (3) the location of the highest densities in IRAS 19124+1106 and IRAS $19132+1035$ on the sides closest to GRS $1915+105$ and, in the case of IRAS $19132+1035$, close to the non-thermal jet, and (4) the bow shock - like structure in the portion of IRAS $19132+1035$ most distant from GRS 1915+105. Some of these lines of evidence are associated only with the southern source. Evidence against 
an association includes (1) the lack of detected high velocity gas at the IRAS sources, (2) the luminosities of the IRAS sources, which are consistent with each of them being powered by one or more hot stars, and (3) the cometary morphology of the northern source, IRAS $19124+1106$, which is common in star forming regions.

Although the physical alignment of the IRAS sources could be a background coincidence, the probability of this is low in view of the small number of IRAS sources, the existence of a jet at IRAS $19132+1035$, and the locations of these sources within the Galaxy. It remains possible that the jet at IRAS $19132+1035$ is an extragalactic background source. This should be tested by radio observations at higher resolution. Because an association between GRS $1915+105$ and these objects may be real we explore in the following the potential characteristics of interactions of ejecta from GRS 1915+105 with the interstellar gas and compare these examples with observations of another somewhat similar source.

\subsection{Production of a large scale cavity}

The angular separation between each IRAS sources and GRS $1915+105$ is $17^{\prime}$. The measured proper motions of the most recent ejections from GRS $1915+105$ are respectively for the approaching and receding ejecta in the interval $\mu_{\text {app }}=[17.6,23.6] \pm 0.5$ mas $/ \mathrm{d}$ and $\mu_{\text {rec }}=[9.0,10.0] \pm$ 0.5 mas/d (Mirabel \& Rodríguez 1994; Fender et al. 1999). This implies typical travel times to reach the lobes of 140 years for the approaching ejecta and of 300 years for the receding ejecta. It is therefore interesting to note that the impact due to the South lobe with the southern IRAS source would appear to occur much earlier than that of the North lobe with the northern IRAS source. Evidence for precession of the jets during the last five years is marginal (Rodríguez \& Mirabel 1999), with the maximum angle no more than 10 degrees. Thus a close alignment of currently observed jets close to GRS $1915+105$ with those from long past outbursts that may be responsible for the IRAS sources may not be a coincidence.

It is perhaps surprising that the separations from GRS $1915+105$ of the two possible interaction zones are identical to better than two percent. These are determined not only by properties of the outflow from GRS $1915+105$, but also by the density and uniformity of the interstellar medium. In order for ejected matter to travel up to 60 pc, a large scale cavity must have been created around GRS 1915+105. Over time this cavity could tend to extend an equal distance in each direction. The probable existence of a cavity around GRS 1915+105 already has been pointed out by Mirabel \& Rodríguez (1996). The plasma clouds ejected during the last few years exhibit ballistic motions (i.e., with no evidence of deceleration), indicating that the density in the interstellar medium through which they have passed is less than 0.05 protons $\mathrm{cm}^{-3}$ (Mirabel \& Rodríguez 1996). These clouds have been ob- served until their emission faded out or became unobservable with the available VLA configuration, typically at distances of one arcsecond from the central source.

Although direct evidence for a cavity is limited to a region very close to GRS $1915+105$, it is likely that the cavity extends well beyond one arcsecond from it. Apart from the two IRAS sources, no phenomena that might be associated with deceleration of recent ejecta have been observed from $1^{\prime \prime}$ angular separation from GRS 1915+105 to $17^{\prime}$. In order to produce a cavity with a linear dimension of many tens of parsecs, even a relativistic source must be active for a much longer period of time than GRS 1915+105 has been observed. From EXOSAT measurements (Reynolds et al. 1999) GRS 1915+105 was active in 1985 but much fainter than it has been since 1992 . For the second discovered superluminal galactic source, GRO J1655-40, Kolb et al. (1997) suggested that the activity of the source is caused by the companion crossing the Hertzsprung gap, a transient phase lasting $\sim 1 \mathrm{Myr}$. The companion of GRS $1915+105$ (Martí et al. 2000) is believed to be more massive than the companion of GRO J1655-40 (Shahbaz et al. 1999), and thus the duration of the active phase would be much shorter, although still sufficiently long, especially since GRS 1915+105 has been much more active than GRO J1655-40.

\subsection{Interaction between ejecta and ambient material}

Considerable heating of the gas must occur at the ends of the cavities, where the ejecta, whether or not partially decelerated en route, impact ambient material. One would expect that much of heating would occur via shock excitation. For the case of GRS $1915+105$ the speed and density of the incident material and the relative amounts of atomic and molecular gas (both in the incident and impacted material) are not known, and thus the spectrum of the shock-heated gas is uncertain. The southern IRAS source shows strong infrared lines of atomic hydrogen and weak infrared line emission from molecular hydrogen, very unlike the emission observed in molecular clouds impacted by protostellar winds having speeds of up to $100 \mathrm{~km} \mathrm{~s}^{-1}$, in which lines of molecular hydrogen dominate and hydrogen recombination lines are nearly non-existent (e.g. Geballe \& Garden 1987).

We note that if the non-thermal feature near the southern lobe is an element of the interaction involving the ejecta of GRS $1915+105$, the collimation of the jet is $f_{\text {coll }}=\frac{\text { length }_{\text {jet }}}{\text { width }_{\text {jet }}}=\frac{15^{\prime}}{7^{\prime \prime}} \gtrsim 100$, ten times larger than the most highly collimated Herbig-Haro objects (see e.g. Bachiller 1996). This implies a jet opening angle of $\lesssim 0.5^{\circ}$ which is consistent with the limit of $<8^{\circ}$ derived by Fender et al. (1999) based on observations of the core, but is more highly constraining. This jet opening angle seems to be in agreement with the jet full opening angle as function of distance from the core for M 87 (Junor et al. 1999), if we take into account the scaling factor between galactic and extragalactic black holes. Therefore, 
as for extragalactic jets, it would require in the galactic case ongoing confinement of the jet at large distances from the source, ruling out free expansion of a relativistic gas.

\section{Induced star formation?}

The similarity of the luminosities of the IRAS sources to those of compact $\mathrm{H}$ II regions containing one or more massive stars, as well as the spectrum of IRAS $19132+1035$, which shows strong hydrogen recombination line emission, suggest the possibility that ejecta from GRS 1915+105 have induced massive star formation at the locations of the IRAS sources, via compression of the interstellar gas. In this scenario the non-thermal radio jet observed at IRAS $19132+1035$ might be interpreted as a Herbig-Haro-like feature, the result of a protostellar wind breaking out of the natal cloud. However, given the length of time for star formation to proceed to this phase, such an explanation would require activity from GRS $1915+105$ at a much earlier time than mentioned above. Moreover, there appears to be no reason for star formation at IRAS $19132+1035$ to produce a close coincidence between the position angle of the radio jet at IRAS $19132+1035$ and that of the line connecting GRS $1915+105$ and IRAS $19132+1035$, as is observed. Finally, the radio emission from Herbig-Haro jets usually is thermal (free-free; Rodríguez 1999). In view of these arguments, we do not regard induced star formation to be a likely explanation for the luminosities of IRAS $19124+1106$ and IRAS $19132+1035$. Dubner et al. (1998) note that in the case of the relativistic jets from SS 433 there is no evidence of induced star formation in the impacted gas.

\subsection{Comparison with SS 433}

In view of the inconclusive evidence linking the IRAS sources with activity originating at GRS $1915+105$, it is perhaps useful to examine other possible examples of such an interaction. Like GRS $1915+105$, the famous X-ray binary SS 433 ejects beams of material at relativistic speeds. This object is located inside the radio shell/supernova remnant W50, which exhibits two lateral extensions with dimensions of tens of parsecs (Dubner et al. 1998). The morphology can be attributed to continuous ejection of magnetic field and high-energy particles from the central source. Sub-arcsecond jets are present at SS 433, with 5 orders of magnitude difference between their extents and those of the far lobes. The power injected into these jets is $\sim 10^{39} \mathrm{erg} \mathrm{s}^{-1}$ according to Dubner et al. (1998), who also estimate that the kinetic energy transferred to the surroundings of SS 433 during the last $\sim 210^{4}$ years amounts to $210^{51} \mathrm{erg}$.

Major ejection events in GRS $1915+105$ are more sporadic than in SS 433, and therefore the average kinetic energy injected into the surroundings over long periods of time may be smaller (Mirabel \& Rodríguez 1999). The kinetic energy of the 1994 March 19 event in GRS $1915+105$ was $\sim 10^{43}$ erg (Rodríguez \& Mirabel 1999). GRS $1915+105$ would need to exhibit one ejection event per hour similar to this one in order to match SS 443. A more likely rate is one event per month (Rodríguez \& Mirabel 1999), in which case $\sim 210^{7}$ years would be required to equal the energy output of SS 443 in $210^{4}$ years. However, we note also that the jets emanating from GRS $1915+105$ always are compact close to the source (Dhawan et al. 2000), which indicates that a continuous injection of energy from GRS $1915+105$ is occuring. In addition to the large events described above, there are also smaller ones, observed at X-ray, radio and infrared wavelengths, where the mechanical luminosities are in the range $10^{37}-10^{39} \mathrm{erg} \mathrm{s}^{-1}$, and where the synchrotron emission is seen up to the infrared wavelengths (e.g. Mirabel et al. 1998; Fender \& Pooley 2000). A comparison of the energetics of GRS 1915+105 with SS 433 and a HerbigHaro object is reported in Table 5 .

Despite the differences between GRS 1915+105 and SS 433, each may have created a cavity of similar linear extent. In view of the uncertainty as to what effects might be observable at the putative ends of the cavity produced by GRS $1915+105$, we have observed the western tip of W50 where the ejecta of SS 443 and the shell remnant interact, in some of the same millimeter lines of ${ }^{12} \mathrm{CO},{ }^{13} \mathrm{CO}$, $\mathrm{H}^{13} \mathrm{CO}^{+}$and $\mathrm{SiO}$ as were observed for the IRAS sources. The observed region in W50 is at the constant declination $05^{\circ} 00^{\prime} 00^{\prime \prime}$ and right ascensions between $19^{\mathrm{h}} 05^{\mathrm{m}} 27^{\mathrm{s}}$ and $19^{\mathrm{h}} 06^{\mathrm{m}} 24^{\mathrm{s}}$ (B1950) (see Dubner et al. 1998, Figs. 1a and $1 \mathrm{~b}$ ). The off position was 15 arcmin east and north of the reference point located at $19^{\mathrm{h}} 06^{\mathrm{m}} 00^{\mathrm{s}} ; 05^{\circ} 00^{\prime} 00^{\prime \prime}$. Measurements at W50 were separated by $24^{\prime \prime}$, with integration times of $5 \mathrm{~min}$ each, except at $(0,0)$ where the integration time was $10 \mathrm{~min}$.

The results are shown in Fig. $12^{9}$ over the relevant velocity range. Emission from ${ }^{12} \mathrm{CO},{ }^{13} \mathrm{CO}$ and $\mathrm{H}^{13} \mathrm{CO}^{+}$ are found roughly $200-300^{\prime \prime}$ west of the reference position, at the westernmost edge of the radio shell (Dubner et al. 1998, Figs. 1a and 1b).

The emission from the high density tracer $\mathrm{H}^{13} \mathrm{CO}^{+}$ is the most compact and probably indicates the location of the strongest interaction. The line profiles of all three species are asymmetric but narrow. SiO 2-1 emission was not detected. These results are very similar to those for IRAS 19132+1035. These similarities between SS 433/W50 and GRS 1915+105/IRAS 19132+1035 and IRAS $19124+1106$ suggest that high density, low velocity molecular clumps may not be unusual products of the interaction of the ejecta of a distant energetic source and the surrounding medium.

\footnotetext{
${ }^{9}$ On the color page at the end of this article.
} 
Table 5. Characteristics of jets from different sources. $\mathrm{HH}=$ Herbig-Haro. The range is the ratio of distance between the far lobes and the subarcsec jets, the collimation factor is the ratio between the length of the jet and its width, and the kinetic power of the ejections is a time average

\begin{tabular}{c|c|c|c}
\hline & $\mathrm{HH}$ & SS 433 & GRS 1915 \\
\hline Velocity & $1-500 \mathrm{~km} \mathrm{~s}^{-1}$ & $0.26 \mathrm{c}$ & $0.92 \mathrm{c}$ \\
Separation & $1 \mathrm{au}-10 \mathrm{pc}$ & $50 \mathrm{pc}$ & $60 \mathrm{pc}$ \\
Range & $10^{6}$ & $10^{5}$ & $10^{4}-10^{5}$ \\
Collimation & $2-10$ & $\geq 12$ & $\sim 100 ?$ \\
Kinetic Pow. & $10^{32} \mathrm{erg} \mathrm{s}^{-1}$ & $10^{39} \mathrm{erg} \mathrm{s}^{-1}$ & $10^{36-39} \mathrm{erg} \mathrm{s}^{-1}$ \\
\hline
\end{tabular}

\section{Conclusions}

We have performed extensive multi-wavelength observations from IR to radio of two radio/IRAS sources axisymetrically located with respect to GRS $1915+105$ and aligned with the position angle of the subarcsec jets. The northern source has the morphology of a common cometary H II region. The observations of the southern cloud reveal a collimated non-thermal structure which may be the far end of a jet of material from GRS $1915+105$. Both sources contain dense clumps of molecular material. Overall the evidence for these regions being interaction zones seems inconclusive. An abbreviated study of what may be a very similar interaction between the ejecta of SS 433 and the surrounding interstellar medium reveals strikingly similar (and also apparently inconclusive) phenomena, perhaps indicating that such phenomena might be produced by sources which via their violent behavior have created large cavities over long periods of time.

Acknowledgements. We thank F. Comeron for allowing us to use part of his time at the ESO/MPI $2.2 \mathrm{~m}$ telescope of the ESO/La Silla. S.C. thanks the astronomers on duty at IRAM, particularly R. Moreno and D. Reynaud, for much assistance and many fruitful discussions during the observations. He thanks also G. Pineau des Forêts and B. Le Floch for helpful discussions. S. C. would like to acknowledge the invitation to work in the dynamic Instituto de Astronomia de Morelia, group of the Universidad Nacional Autonoma de Mexico (UNAM), and the fruitful discussions leading to some of the ideas in this paper. He thanks also the Groupe de Recherche Accrétion-Disque-Jets (GdR ADJ) of the French Centre National de la Recherche Scientifique (CNRS) altogether with the UNAM for the financial support of this journey. We thank R. P. Fender for pointing out to us the previous mislabelling of the region $\mathrm{G} 45.45+0.06$. We also thank the anonymous referee for prompt and useful comments, which allowed us to improve the manuscript. S. C. acknowledges support from grant F/00-180/A from the Leverhulme Trust. I. F. M. acknowledges support from CONICET/Argentina. The UK Infrared
Telescope is operated by the Joint Astronomy Centre on behalf of the UK Particle Physics and Astronomy Research Council. The ISOCAM data presented in this paper were analysed using "CIA", a joint development by the ESA Astrophysics Division and the ISOCAM Consortium.

\section{References}

Bachiller, R. 1996, ARA\&A, 34, 111

Brand, J., \& Blitz, L. 1993, A\&A, 275, 67

Castro-Tirado, A. J., Brandt, S., Lund, N., et al. 1994, ApJS, 92, 469

Cesarsky, C. J., Abergel, A., Agnese, P., et al. 1996, A\&A, 315, L32

Chaty, S. 1998, Ph.D. Thesis, University Paris XI

Chaty, S., Mirabel, I. F., Duc, P.-A., Wink, J. E., \& Rodríguez, L. F. 1996, A\&A, 310, 825

Chaty, S., Rodríguez, L. F., \& Mirabel, I. F. 2000, in A. Letters and Communications (eds.), Proceedings of X-ray Astronomy '999 - Stellar Endpoints, AGN and the Diffuse Background, ed. G. Malaguti, G. Palumbo \& N. White (Gordon \& Breach Singapore), in press

Denoyer, L. K. 1979, ApJ, 232, L165

Dhawan, V., Mirabel, I., \& Rodríguez, L. 2000, ApJ, 543

Downes, D., Wilson, T., Bieging, J., \& Wink, J. 1980, A\&AS, 40, 379

Dubner, G., Holdaway, M., Goss, M., \& Mirabel, I. F. 1998, A\&AS, 116, 1842

Feldt, M., Stecklum, B., Henning, T., et al. 1998, A\&A, 339, 759

Fender, R. P., Garrington, S. T., McKay, D. J., et al. 1999, MNRAS, 304, 865

Fender, R. P., \& Pooley, G. G. 2000, MNRAS, 318, L1

Geballe, T. R., \& Garden, R. 1987, ApJ, 317, L107

Junor, W., Biretta, J. A., \& Livio, M. 1999, Nature, 401, 891

Kolb, U., King, A., Ritter, H., \& Frank, J. 1997, ApJ, 485, L33

Martí, J., Mirabel, I. F., Chaty, S., \& Rodríguez, L. F. 2000, A\&A, 356, 943

Mirabel, I. F., \& Rodríguez, L. F. 1999, ARA\&A, 37, 409

Mirabel, I. F., Dhawan, V., Chaty, S., et al. 1998, A\&A, 330, L9

Mirabel, I. F., \& Rodríguez, L. F. 1994, Nature, 371, 46

Mirabel, I. F. and Rodríguez, L. F. 1996, in Solar and Astrophysical Magnetohydrodynamic Flows ed. K. Tsinganos (Kluwer Academic Publishers), 683

Reynolds, A. P., Parmar, A. N., Hakala, P. J., et al. 1999, A\&AS, 134, 287

Rodríguez, L. F. 1999, in Star Formation 1999, Proceedings of Star Formation 1999, ed. N. R. O. T. Nakamoto, held in Nagoya, Japan, June 21-25, 1999, 257

Rodríguez, L. F., \& Mirabel, I. F. 1998, A\&A, 340, L47 (RM98)

Rodríguez, L. F., \& Mirabel, I. F. 1999, A\&J, 511, 398

Shahbaz, T., van der Hooft, F., Casares, J., Charles, P., \& van Paradijs, J. 1999, MNRAS, 306, 89

Starck, J. L., Abergel, A., Aussel, H., et al. 1999, A\&AS, 134, 135 


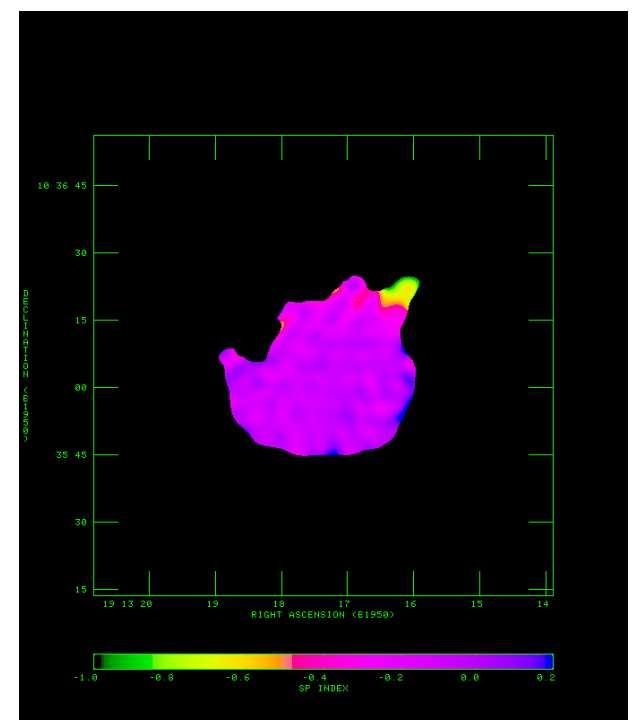

Fig. 3. Spectral index map of IRAS $19132+1035$ made from the 20 and $6-\mathrm{cm}$ maps. The color coding for the spectral index is given at the bottom of the figure. Note the negative, nonthermal spectral index of the jet feature to the northwest
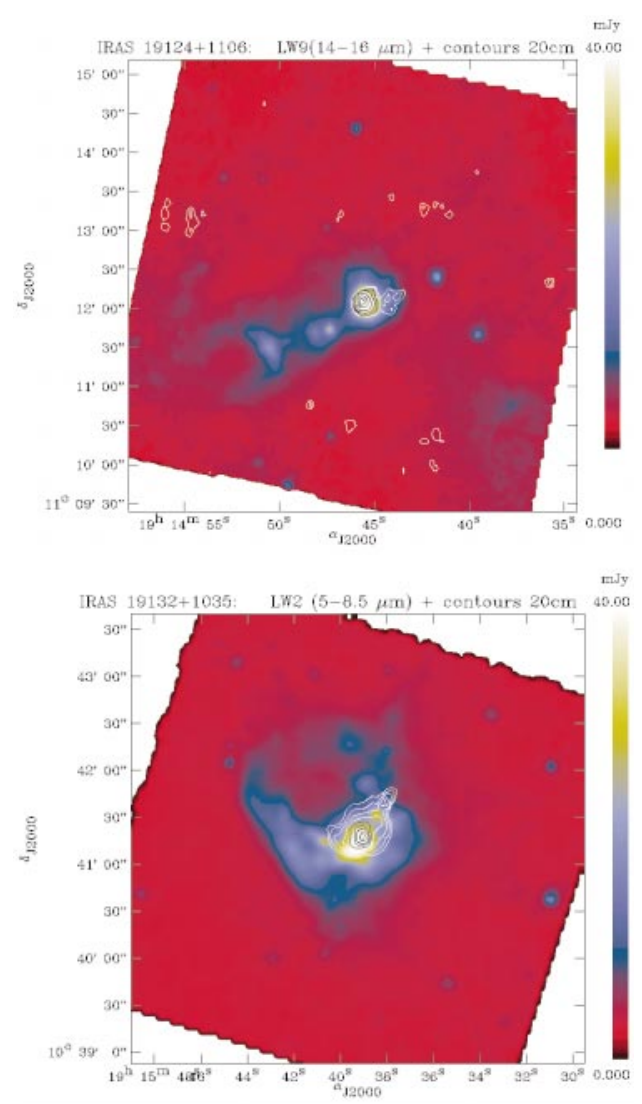

Fig. 6. Top: ISO map of the source IRAS 19124+1106, at $\lambda=$ $15 \mu \mathrm{m}$, taken with the LW9 filter. Superimposed are the $20 \mathrm{~cm}$ radio contours at $0.4,0.6,1,2,5,10$ and $15 \mathrm{mJy}$. Bottom: ISO map of the source IRAS $19132+1035$, at $\lambda=7 \mu \mathrm{m}$, taken with the LW2 filter. Superimposed are the $20 \mathrm{~cm}$ radio contours at the levels $0.2,0.4,0.7,1.2,1.8,2.5,3,4$ and $5 \mathrm{mJy}$
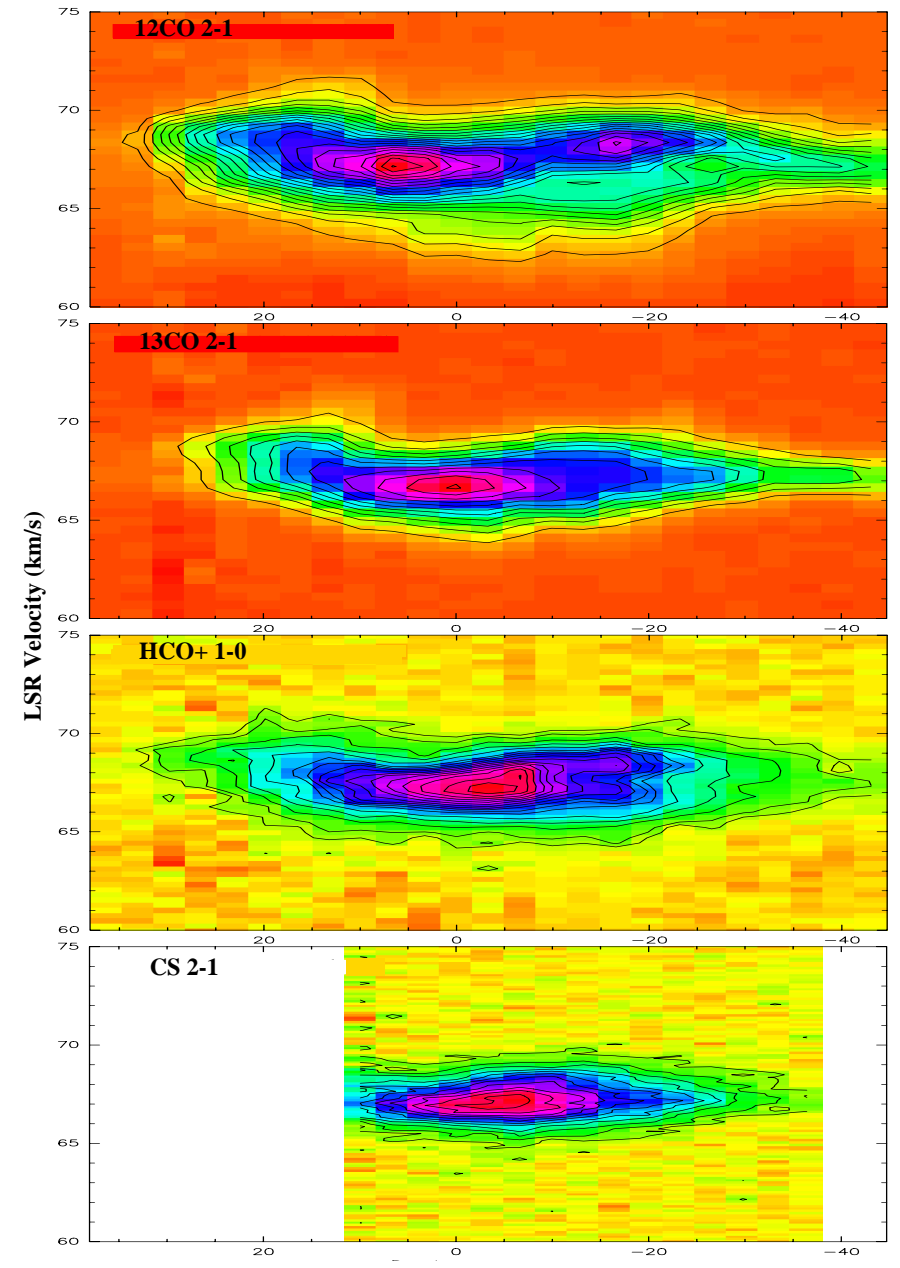

RA Offset (arcsec)

Fig. 7. Observations of IRAS $19132+1035$. Offsets are relative to the position of maximum radio emission observed at the VLA. The black contours are antennae iso-temperature: ${ }^{12} \mathrm{CO}$ : $T_{\mathrm{A}}^{*}=-1$, and from 1 to $20 \mathrm{~K}$ separated by an interval of $1 \mathrm{~K}$; ${ }^{13} \mathrm{CO}: T_{\mathrm{A}}^{*}=-1$, and from 1 to $11 \mathrm{~K}$ separated by an interval of $1 \mathrm{~K} ; \mathrm{H}^{13} \mathrm{CO}^{+}: T_{\mathrm{A}}^{*}=-1$, and from 0.2 to $2.1 \mathrm{~K}$ separated by an interval of $0.1 \mathrm{~K}$; CS: $T_{\mathrm{A}}^{*}=-1$, and from 0.2 to $2.2 \mathrm{~K}$ separated by an interval of $0.2 \mathrm{~K}$ 

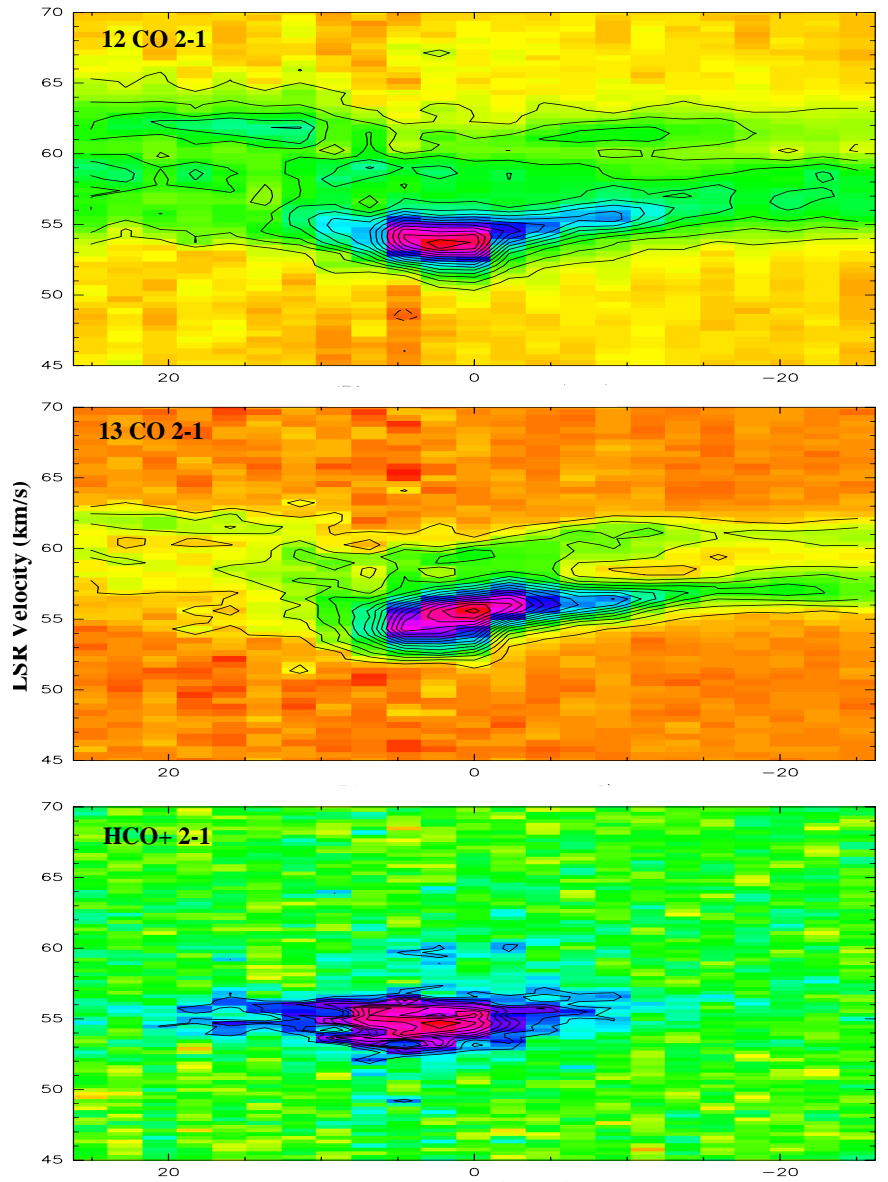

RA Offset (arcsec)

Fig. 10. Observations of IRAS $19124+1106$. Offsets are relative to the position of maximum radio emission observed at the VLA. The black contours are antennae iso-temperatures: ${ }^{12} \mathrm{CO}: T_{\mathrm{A}}^{*}=-1$, and from 1 to $14 \mathrm{~K}$ separated by an interval of $1 \mathrm{~K} ;{ }^{13} \mathrm{CO}:-1$ and from 0.5 to $8 \mathrm{~K}$ separated by an interval of $0.5 \mathrm{~K} ; \mathrm{H}^{13} \mathrm{CO}^{+}$: -10.30 .4 and from 0.5 to $0.8 \mathrm{~K}$ separated by an interval of $0.05 \mathrm{~K}$
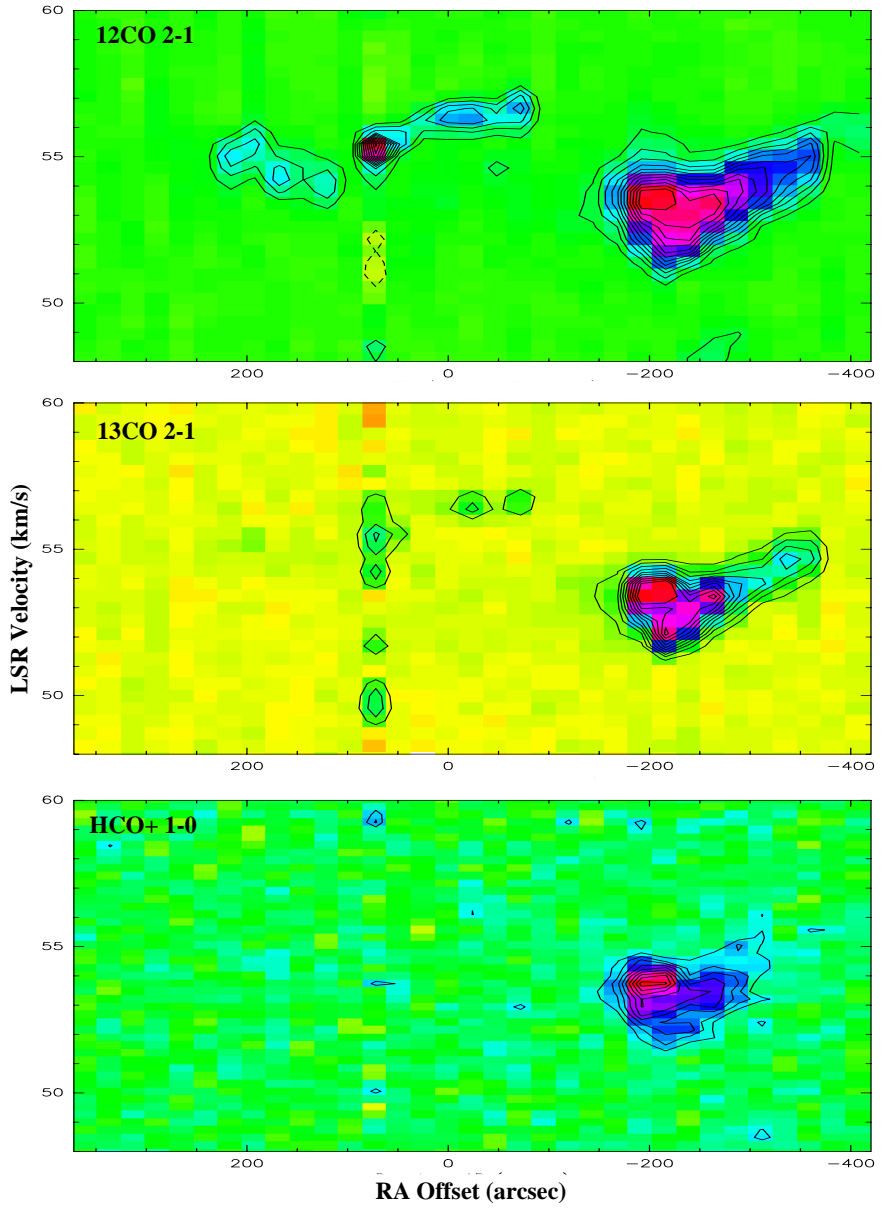

Fig. 12. Observations of W50, the supernova remnant shell surrounding SS 433. Offsets are relative to the position of the central source. The black contours are antennae isotemperatures: ${ }^{12} \mathrm{CO}: T_{\mathrm{A}}^{*}=-1$ and from 1 to $12 \mathrm{~K}$ separated by $1 \mathrm{~K} ;{ }^{13} \mathrm{CO}:-1$ and from 0.5 to $5 \mathrm{~K}$ separated by $1 \mathrm{~K} ; \mathrm{H}^{13} \mathrm{CO}^{+}$: -1 and from 0.2 to $1 \mathrm{~K}$ separated by $0.1 \mathrm{~K}$ 